\title{
Evolución de tres índices bioclimáticos para la vid en San Martín y Luján de Cuyo (Mendoza, Argentina)
}

\author{
Martín Cavagnaro ${ }^{1, a}$, Pablo Canziani ${ }^{2,3}$, José Portela ${ }^{4}$ y Walter Robledo ${ }^{3,5}$ \\ ${ }^{1}$ Dirección de Agricultura y Contingencias Climáticas, Gobierno de Mendoza, Mendoza, Argentina \\ 2 PEPACG (Equipo Interdisciplinario para el Estudios de Procesos Atmosféricos en el Cambio Global), Pontificia \\ Universidad Católica Argentina, Ciudad Autónoma de Buenos Aires, Buenos Aires, Argentina \\ ${ }^{3}$ CONICET (Consejo Nacional de Investigaciones Científicas y Técnicas), Argentina \\ ${ }^{4}$ INTA (Instituto Nacional de Tecnología Agropecuaria), EEA La Consulta, San Carlos, Mendoza, Argentina \\ ${ }^{5}$ Facultad de Ciencias Agropecuarias, Universidad Nacional de Córdoba, Córdoba, Argentina
}

\begin{abstract}
Resumen. La Región de Cuyo, Argentina, se caracteriza por su clima templado árido a semiárido, apropiado para el cultivo irrigado de la vid. La Provincia de Mendoza representa cerca del 70\% del área nacional cultivada con vid, con una producción superior al 65\%. Los departamentos San Martín y Luján de Cuyo son reconocidos por su alta producción vitícola y la calidad enológica de sus uvas, respectivamente. Son escasos aún los estudios sobre el efecto de variaciones climáticas sobre las respuestas de los viñedos, principalmente en contexto de cambio climático, sumado a la problemática de la disposición de cantidad suficiente de registros. El presente trabajo se propone estudiar la evolución de tres índices bioclimáticos para la vid, y su potencial influencia en los cambios de respuesta en la marcha fenológica durante cada temporada productiva. Se infiere que estas localidades están experimentando un cambio térmico, con sus potenciales cambios en los rendimientos, composición de bayas y marcha fenológica anual.
\end{abstract}

\section{Introducción}

El clima en la región centro-oeste de la Argentina, situada entre $28^{\circ}-36^{\circ} \mathrm{S}$ y $65^{\circ}-70^{\circ} \mathrm{W}$, conocida como la región de Cuyo, es templado árido a semiárido, de gran heliofanía, escasas lluvias y suelos aluvionales, propios para el cultivo de la vid con irrigación de sus oasis cultivados con agua proveniente del deshielo de la Cordillera de Los Andes, ya que las precipitaciones promedio en la región están en el orden de los 200 a 300 mm anuales, insuficientes para la agricultura. La provincia de Mendoza presenta condiciones de agroclimáticas favorables para el cultivo de la vid por irrigación artificial. Cerca del $70 \%$ del área nacional cultivada con vid se encuentra en esta provincia, que tiene una producción superior al $65 \%$ del total nacional, siendo la producción de uvas y su industrialización componentes muy importantes de la economía regional.

El departamento San Martín, ubicado en la Zona Este de Mendoza es reconocido por su alta producción vitivinícola. La superficie cultivada con vid se ha incrementado en la última década llevando a un crecimiento de la industria vitivinícola argentina sin precedentes. El departamento de Luján de Cuyo pertenece a la Zona Centro de la Provincia de Mendoza (Argentina), ubicada junto al departamento Maipú en la denominada "Primera Zona", siendo una de las subregiones más destacadas a nivel internacional por la calidad de sus uvas blancas y tintas de alta calidad enológica la calidad de sus tierras y condiciones microclimáticas.

\footnotetext{
${ }^{a}$ Autor de correspondencia: martin_cavagnaro@hotmail.com
}

En diferentes trabajos $[15,17,20]$ se muestra la influencia del clima y su variabilidad en la duración del ciclo fenológico y en sus diferentes etapas, con una tendencia a su modificación, de acuerdo a las diferentes zonas productivas. Los tenores azucarinos también se han ido incrementando, logrando cada vez más concentraciones de azúcares reductores. Desde hace varios años se busca en las uvas no sólo la madurez industrial, sino la "madurez fenólica", sobre todo en uvas tintas, resultando cosechas con mayores tenores azucarinos y vinos muy alcohólicos.

Se ha demostrado que cerca del $50 \%$ de la variación interanual en producción total de vid en Mendoza está determinada por las variaciones interanuales de la precipitación y la temperatura dentro del período 1979$2009[1-3,7]$.

La cantidad y calidad de producción depende del desarrollo del ciclo de la vid, el cual depende de las condiciones ambientales y nutricionales del ciclo anual. Desde hace muchos años se intenta lograr un acercamiento hacia la comprensión de las relaciones del cultivo de la vid y su respuesta al clima y su variabilidad [8-11,19,21,22].

Estudios realizados en otras áreas vitivinícolas del mundo sugieren que la variabilidad climática generada por el calentamiento global, puede producir cambios significativos en el ciclo fenológico de la vid [5, 14-17, 20].

En este sentido se toman como herramienta de trabajo las proyecciones de escenarios climáticos obtenidas a través de modelos de circulación general (MCG) según los posibles escenarios socio-económicos planteados por el IPCC [12]. Estas proyecciones se utilizan para examinar

This is an Open Access article distributed under the terms of the Creative Commons Attribution License 4.0, which permits unrestricted use, distribution, and reproduction in any medium, provided the original work is properly cited. 
cómo los sistemas pueden responder a estos cambios [13]. Diversas investigaciones demuestran que puede haber cambios en las fronteras de la geografía vitícola, infiriendo que pueden producirse modificaciones en términos de calidad debido al clima $[6,15,16]$. En su trabajo de 2008, Barbero y otros [5] observaron cambios en las zonificaciones potenciales de la Patagonia Argentina. Asimismo existen algunas aproximaciones en cuanto a las relaciones de la fenología de la vid en algunas variedades y la influencia que en ella ejerce la variabilidad térmica.

Sin embargo, para poder estimar posibles proyecciones futuras en la actividad vitivinícola es importante saber cual es la respuesta a la variabilidad y tendencia presente de diversos indicadores relacionados con la producción de uvas y la elaboración del vino. Para este fin es importante evaluar cómo es esa respuesta.

Se intenta establecer en este trabajo la evolución de tres de los más importantes y más utilizados índices bioclimáticos utilizados para la vid y su potencial influencia en los ya mencionados cambios en las respuestas de su marcha fenológica en cada temporada productiva.

\section{Materiales y método}

Se trabajó calculando tres índices bioclimáticos clásicos para el cultivo de la vid: Integral Térmica de Amerine y Winkler (IW), Índice de Huglin (IH) e Índice de Frescor Nocturno (IFN), cuyas fórmulas son las siguientes:

Integral Térmica de Amerine y Winkler [22]:

$$
I W=\sum(T m d-10)
$$

donde Tmd es la temperatura media diaria desde el $1^{\circ}$ de octubre al 30 de abril

Índice de Huglin [11]:

$$
I H=\sum[(\operatorname{Tmax}-10)+(\text { Tmed }-10)]^{1 / 2},
$$

donde

Tmax es la temperatura máxima diaria desde el $1^{\circ}$ de setiembre al 31 de marzo

Tmed es la temperatura media diaria desde el $1^{\circ}$ de setiembre al 31 de marzo

$\underline{\text { Índice de Frescor Nocturno }}$ [21]

$$
\text { IF } N=\text { Tminmmarzo, }
$$

donde Tminmmarzo es la temperatura mínima media del mes de marzo

Se utilizó la base de datos de temperaturas mínimas, máximas y medias diarias de la estación San Martín $\left(33^{\circ} 46^{\prime} \mathrm{S}, 68^{\circ} 20^{\prime} \mathrm{W}, 653 \mathrm{msnm}\right)$ del Servicio Meteorológico Nacional, en el período comprendido entre los años 1957 a 2009. También, para efectuar los mismos cálculos se tomó la base de datos de temperaturas máximas, mínimas y medias diarias de la estación Chacras de Coria (32 59’ S, 68 $50^{\prime}$ W, 921 msnm) de la Facultad de Ciencias Agrarias de la Universidad Nacional de Cuyo.

Se trabajó con paquete estadístico InfoStat 7 y con herramientas Microsoft Office Excel 2003, donde se calcularon los índices de regresión lineal y se elaboraron

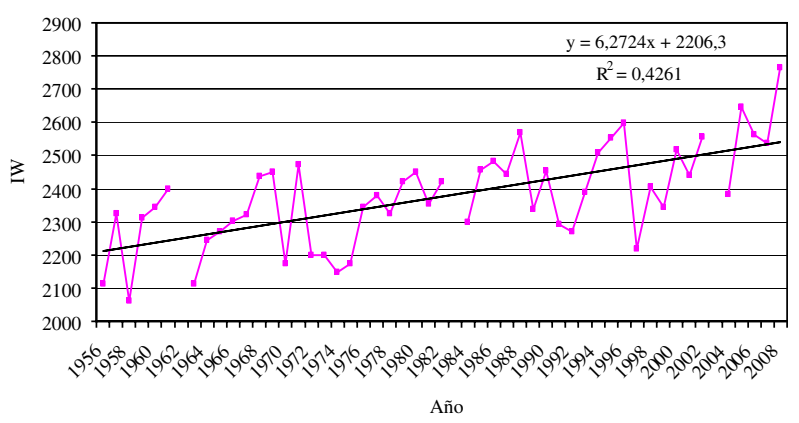

Figura 1. Evolución anual del IW para la localidad San Martín, considerando el período 1957-2008.

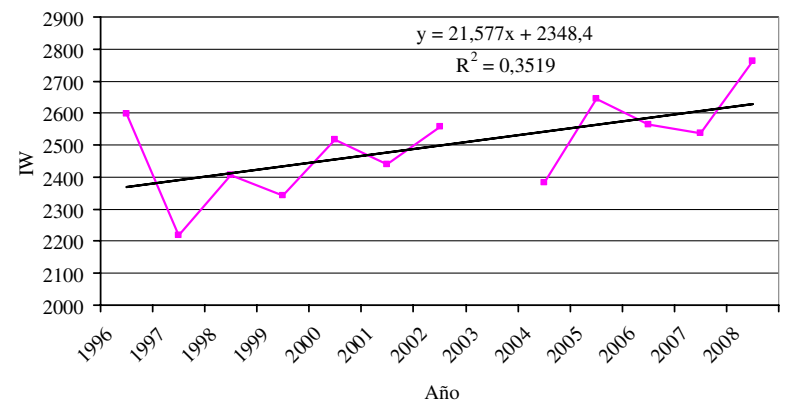

Figura 2. Evolución anual del IW para la localidad San Martín, considerando el sub-período 1996-2008.

los gráficos correspondientes a las evoluciones anuales y períodos decádicos de cada índice para cada una de las locaciones.

\section{Resultados y discusión}

\subsection{Departamento San Martín}

La evolución anual del Índice de Winkler (IW) muestra, para todo el período considerado, una tendencia significativa y positiva de 6,27 unidades del índice $(\mathrm{p}=0,0001)$ para un $95 \%$ de confianza, aunque mostrando (Fig. 1) un coeficiente de determinación bajo $\left(\mathrm{R}^{2}=0.42\right)$. Esto da, en principio una idea de un aumento muy gradual para el período considerado de este índice, mostrando en el último subperiodo (1996 a 2008) su mayor tendencia $(21,58$ unidades de IW, $\mathrm{p}=0.042, \alpha=0.05$ ) de crecimiento del mismo (Fig. 2).

En cuanto al Índice de Huglin, en la evolución anual para esta localidad se observa también una tendencia significativa $(\mathrm{p}=0.0001$ para un $\alpha=0,05)$ de 4.64 unidades de IH, aunque mostrando (Fig. 3) un coeficiente de determinación bajo $\left(\mathrm{R}^{2}=0.27\right)$. Esto da, en principio una idea de un aumento muy gradual para el período considerado de este índice.

Rusticucci y Barrucand [18] destacan que en territorio nacional de Argentina el número de noches frías por temporada indica claramente un menor número de ocurrencias por el verano; de manera similar, el número de noches cálidas tiende a aumentar, especialmente en la región noreste.

Realizado el análisis del Índice de Frescor Nocturno (IFN), se ve claramente la evolución (Fig. 4 y Tabla 1) 


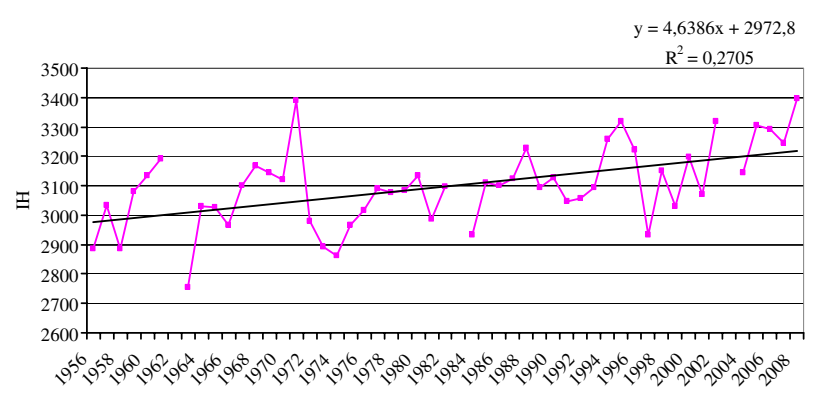

Año

Figura 3. Evolución anual del IH para la localidad San Martín, considerando el período 1957-2008.

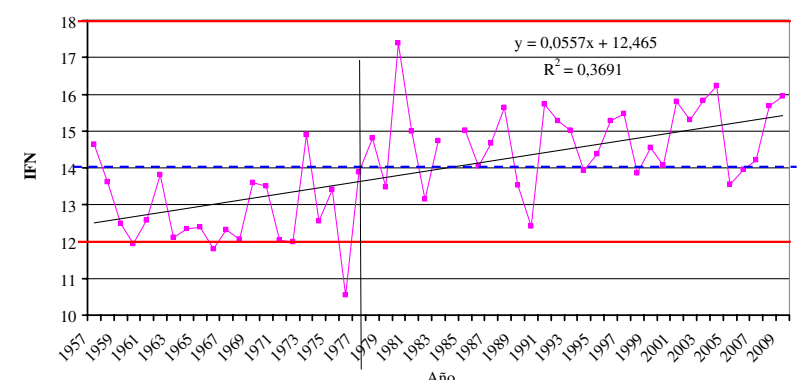

Figura 4. Evolución anual del IFN para la localidad San Martín, considerando el período 1957-2009.

Tabla 1. Categorías de clasificación del Índice de Frescor Nocturno (Tonietto y Carbonneau, 2004).

\begin{tabular}{|c|c|c|}
\hline Clase & Intervalo & Nombre de la clase \\
\hline IF+2 & $<12^{\circ} \mathrm{C}$ & NOCHES MUY FRÍAS \\
\hline IF+1 & 12 a $14^{\circ} \mathrm{C}$ & NOCHES FRÍAS \\
\hline IF-1 & 14 a $18^{\circ} \mathrm{C}$ & NOCHES TEMPLADAS \\
\hline IF-2 & $>18^{\circ} \mathrm{C}$ & NOCHES CÁLIDAS \\
\hline
\end{tabular}

hacia noches cada vez menos frescas, con una tendencia creciente positiva y significativa $(0.056$ IFN) para un $\alpha=0.05(\mathrm{p}<0.0001)$, pudiendo observar la evolución intradecádica del IFN en esta región.

\subsection{Departamento Luján de Cuyo}

La evolución anual del Índice de Winkler (IW) muestra, en este caso, una tendencia significativa y positiva de 4,06 unidades de IW ( $\mathrm{p}=0.0005)$ para un $95 \%$ de confianza, aunque mostrando (Fig. 5) un coeficiente de determinación bajo $\left(\mathrm{R}^{2}=0.21\right)$.

En cuanto al Índice de Huglin, en la evolución anual para Luján de Cuyo se observa también una tendencia de 4.06 IW significativa $(\mathrm{p}=0.0001)$ para un $95 \%$ de confianza, aunque mostrando (Fig. 6) un coeficiente de determinación bajo $\left(\mathrm{R}^{2}=0.26\right)$. La tendencia para el período 1996-2008 (Fig. 7) muestra una diferencia importante, ya que la tendencia es de 0,45 unidades de IW, con un coeficiente $\mathrm{R}^{2}$ mucho más bajo que para San Martín $\left(\mathrm{R}^{2}=0.0001\right)$ y no estadísticamente significativa.

Analizando el Índice de Frescor Nocturno (IFN), se ve claramente la evolución hacia noches cada vez menos frescas (Fig. 8), con una tendencia creciente (en menor

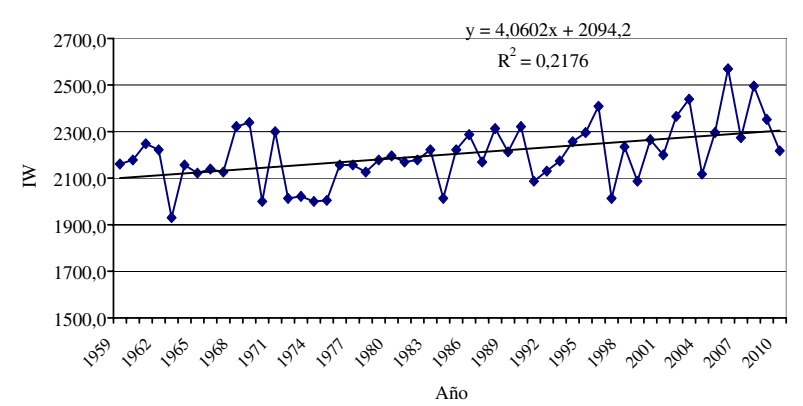

Figura 5. Evolución anual del IW para la localidad Luján de Cuyo, considerando el período 1959-2010.

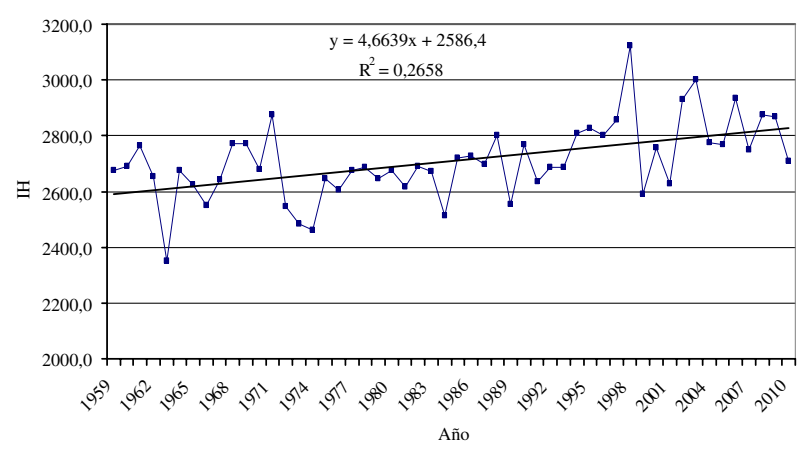

Figura 6. Evolución anual del IH para Luján de Cuyo, considerando el período 1959-2010.

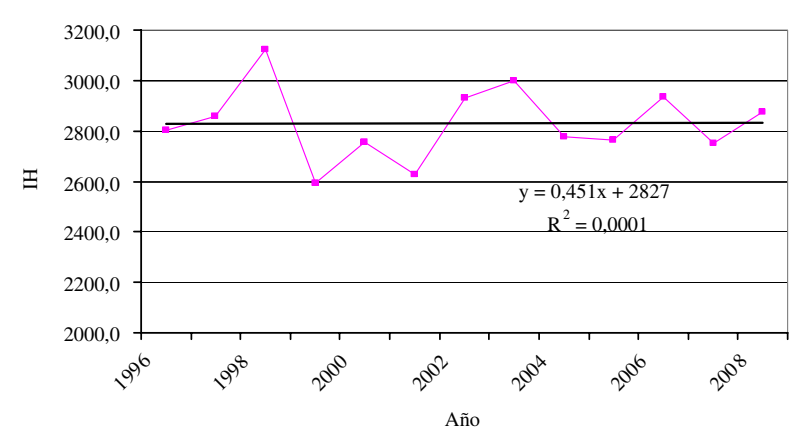

Figura 7. Evolución anual del IW para la localidad Chacras de Coria, considerando el sub-período 1996-2008.

grado que lo visto en San Martín) positiva y significativa de 0.033 IFN para un $\alpha=0.05$ ( $\mathrm{p}<0.003$ ).

Teniendo en cuenta los resultados obtenidos, puede decirse, de manera general, que en ambas localidades, los índices tienen una tendencia hacia valores más altos, considerando los respectivos períodos considerados. Es de destacar que las mayores pendientes de aumento se obtuvieron para San Martín, aunque para Luján de Cuyo siguen siendo estadísticamente significativas.

Se observa que, en ambas estaciones, para los índices de Winkler y de Huglin, la variabilidad interanual desde mediados de la década de 1990 es mayor que en gran parte de los años previos. No se observa lo mismo para el IFN ya que se observa en ambas estaciones y a lo largo de toda la muestra, variabilidad interanual significativa.

Resulta más visible el análisis realizado sobre períodos decádicos, donde estas tendencias se muestran mucho más marcadas que en el análisis de variación interanual. Las tendencias de aumento de estos índices tienen como 


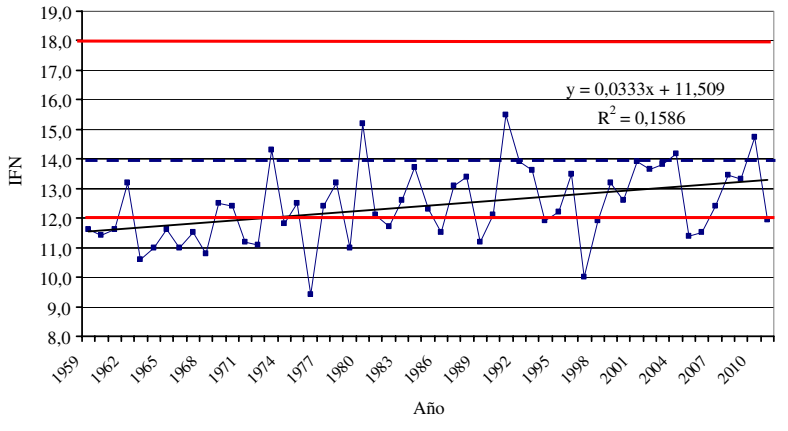

Figura 8. Evolución anual del IFN para Chacras de Coria, considerando el período 1959-2010.
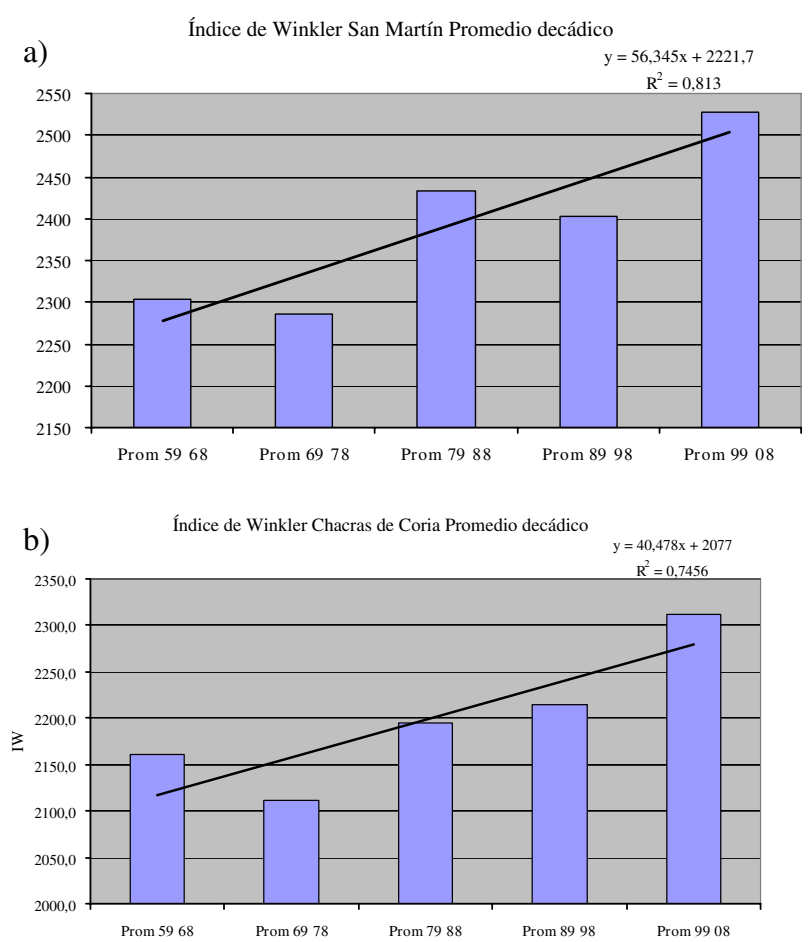

Figura 9. Evolución decádica del IW para las localidades San Martín (a) y Chacras de Coria (b).

principal causa a la temperatura del aire en la estación de crecimiento estival de la vid en esta latitud.

En el caso de los índices IW e IH, las probables consecuencias de estos aumentos tienen que ver con una respuesta hacia mayores tasas fotosintéticas y cambios en la actividad fisiológica de los cultivos que pudiesen generar alteraciones en la fenología de los mismos.

El análisis de estos índices, tomando promedios en forma decádica muestra un aumento mucho más marcado que en la series anuales en todos los casos (Figs. 9, 10 y 11).

\section{Conclusiones}

Tanto San Martín como Luján de Cuyo están experimentando un cambio térmico, según lo observado a lo largo del período en estudio, considerando las tendencias de aumento de los índices calculados (IW, IH e IFN), pudiendo observarse que la mayor magnitud y tendencia de aumento se produce en San Martín, atendiendo a
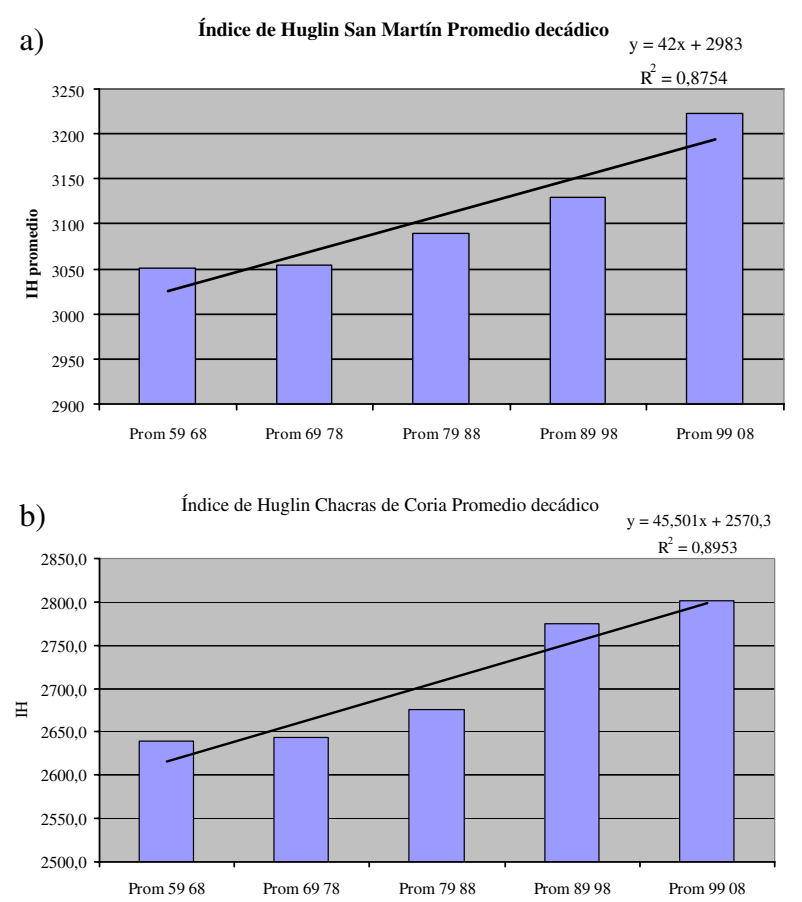

Figura 10. Evolución decádica del IH para las localidades San Martín (a) y Chacras de Coria (b).
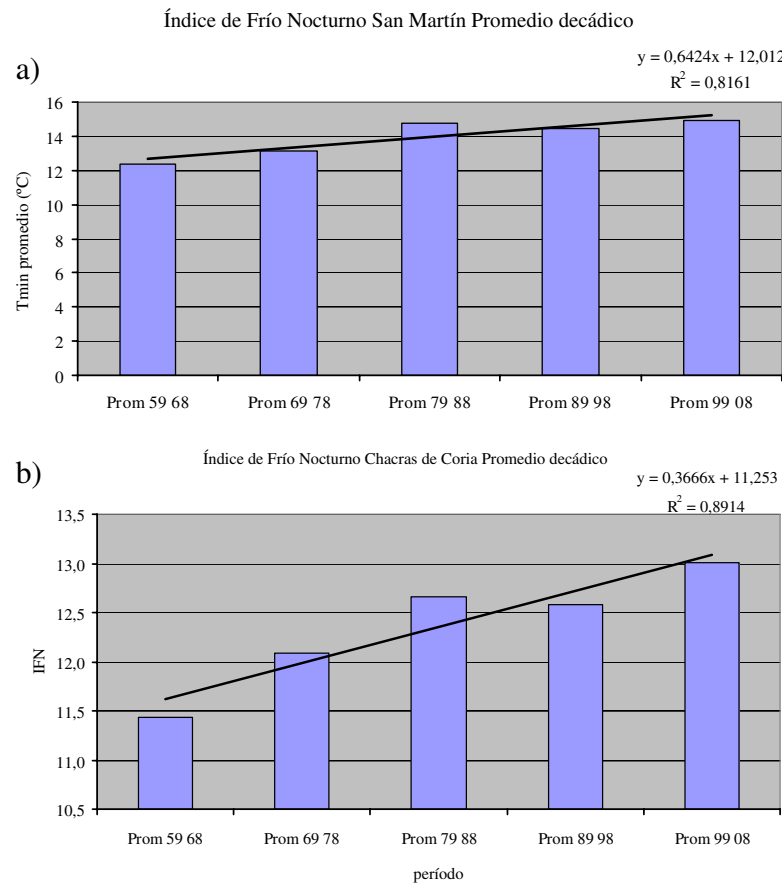

Figura 11. Evolución decádica del IFN para las localidades San Martín (a) y Chacras de Coria (b).

su ubicación geográfica, al cual presenta naturalmente menores amplitudes térmicas diarias y mayores temperaturas diurnas. La tendencia en aumento en ambas localidades de la temperatura de las noches en época de madurez de las uvas, puede traer aparejados algunos potenciales cambios de respuesta en el rendimiento de los cultivos de vid para ambas localidades, así como en la composición fisico-química de las bayas debido al 
escaldado de los racimos evitando el normal desarrollo de los precursores aromáticos, y en la marcha fenológica anual de éstos.

Es necesario, por tanto, trabajar con prácticas de campo que minimicen los efectos negativos en términos de rendimiento y calidad.

\section{Referencias}

[1] E. Agosta, P. Canziani and M. J. App. Meteorol. Clim. J. Appl. Meteor. Clim, doi:10.1175/JAMC-D11-0165.1. (2012)

[2] E. Agosta, M. Cavagnaro y P. Canziani. Enología, ISSN 1668-3889, N² 2, Año VII, 12 pp. (2010)

[3] E. Agosta. Tesis de Doctorado, Fac. Cs. Exactas y Naturales, UBA, 178 pp. (2006)

[4] M. Amerine and A. Winkler. Hilgard 15: 493-673. (1944)

[5] N. Barbero, C. Rossler y P. Canziani. Enología, 5(2), 8 pp. (2008)

[6] R, Butterfield, M. Gawith, P. Harrison, K. Lonsdale and J. Orr, Environmental Change Institute, University of Oxford. (2000)

[7] M. Cavagnaro y E. Agosta. CONGREMET XI (2012)

[8] C. Fregoni and S. Pezzutto. Progr. Agric. Vitic. 18: 390-396. (2000)

[9] J. Glastones. Viticulture and Environment, ISBN 1875130-12-8, 310 pp. (1992)
[10] J. Gladstones Wine, terroir and climate change ISBN: 978186254924 1, 288 pp. (2011)

[11] P. Huglin. Comptes Rendus de l'Académie de l'Agriculture de France 64: 1117-1126. (1978)

[12] IPCC. Summary for Policymakers, Climate Change 2013: The Physical Science Basis. Cambridge University Press. (2013)

[13] G. Jones. Whitman College Economics Department. Working Paper, $\mathrm{N}^{\circ}$ 7. (2007)

[14] G. Jones, E. Duchene, D. Tomasi, J. Yuste, O. Braslavksa, H. Schultz, C. Martinez, S. Boso, F. Langellier, C. Perruchot, and G. Guimberteau. GESCO 2005. (2005)

[15] G. Jones and R. Davis. Am. J. of Enology and Viticulture, 51(3), 249-261. (2000)

[16] G. Kenny and P. Harrison. J. of Wine Research, 3, 163-183. (1992)

[17] M. McCarthy. Aust J Grape Wine Res 5, 10-16. (1999)

[18] M. Rusticucci and M. Barrucand. J. Climate, 17, 4099-4107. (2004)

[19] F. Santibáñez. Tópicos de actualización en viticultura y enología. (2002)

[20] H. Schultz. Aust J Grape Wine Res 6: 2-12 (2000)

[21] J. Tonietto and A. Carbonneau. Agricultural Forest Meteorology, 124: 81-97. (2004)

[22] A. Winkler, J. Cook, W. Kliewer and L. Lider. General viticulture. (1974) 